\title{
Laser Induced Periodic Surface Structures on Polymer Films: From Fundamentals
}

\section{to Applications.}

Esther Rebollar $^{*}{ }^{*}$, Marta Castillejo ${ }^{1}$, Tiberio A. Ezquerra ${ }^{2}$

${ }^{1}$ Instituto de Química Física Rocasolano, IQFR-CSIC, Serrano 119, 28006 Madrid, Spain

${ }^{2}$ Instituto de Estructura de la Materia, IEM-CSIC, Serrano 121, 28006 Madrid, Spain

*Corresponding author. E-mail: e.rebollar@iqfr.csic.es

\begin{abstract}
The generation of nanostructured polymer films has been a challenge during the last decades. Surface nanostructuring based on the imprinting of nanoscale patterns on a homogeneous surface or on the deposition of nanostructures on the surface serves advantageously for nanofabrication of functional polymer materials. Advanced nanolithography typically requires multiple-steps procedures involving clean-room facilities, high vacuum or complex mask fabrication. Alternatively, laser-based methods enable high spatial resolution patterning of soft polymeric matter and afford the sought versatility and reliability without the need of stringent ambient conditions. In particular, the technique of laser induced periodic surface structures (LIPSS) has been successfully applied to nanostructuring of polymer films using a polarized laser source at several laser wavelengths and pulse durations. In this paper the formation of LIPSS on polymer films will be described. In particular, the possibilities of tuning the period and shape of the structures will be discussed since the control of the characteristics of the superficial structures can be crucial in order to match the requirements of a particular application. Additionally, an overview about the main and potential applications of LIPSS in
\end{abstract}


polymer films is provided. In particular, the use of rippled polymer films as substrates for cell culture/alignment, surface enhanced Raman scattering sensors, or applications in photovoltaics will be reviewed.

Keywords: Laser induced periodic surface structures, polymer thin films, atomic force microscopy, semiconducting polymers, cell culture 


\section{Contents}

1. Introduction

2. Laser nanostructuring of polymer films

2.1. LIPSS formation in polymers using nanosecond laser pulses

2.2. In situ monitoring of LIPSS formation

2.3. Different approaches for the formation of alternative periodic structures

2.3.1. LIPSS with different periods: variation of incidence angle

2.3.2. LIPSS with different shapes: variation of polarization

2.4. LIPSS formation in polymers using femtosecond laser pulses

2.5. Physico-chemical modifications upon LIPSS formation

3. Applications of polymer LIPSS

3.1. Polymer LIPSS for cell culture

3.2. Polymer LIPSS for Surface Enhanced Raman Spectroscopy (SERS) substrates

3.3. LIPSS on semiconducting polymers

4. Conclusion and outlook

Acknowledgements

References 


\section{Introduction}

The surface properties of polymers are of crucial importance in order to provide certain chosen functionalities. In general, nanofabrication allows the manipulation of nanoscale building blocks for a desired purpose by furnishing macroscopic materials with nanometer-scale structural motifs that confer modified physical and chemical properties. Among the most popular examples, the hierarchical structure of the gecko foot or of the lotus leave are paradigmatic cases of an almost universal natural adherence [1] and of a natural superhydrophobic [2] behavior respectively. It therefore appears to be a rewarding project to structure the surface of a polymeric material periodically by varying both pattern and length scales. The periodic surface structures thus obtained can then be used as substrates in many fields stranding from organic photonics and microelectronics to biomedicine [3-7]. These substrates are generally provided as either spin-coated or free-standing polymer films.

Common techniques for generating polymer structures at the nanoscale are mainly based in soft lithography methods, like micro-contact printing or nanoimprint lithography (NIL) among others [4, 8-11]. These methods aim at reproducibility and low-cost, and generally provide versatile processing strategies. Lithographic procedures suffer from limitations on spatial resolution. An additional drawback is the need of multiple-steps procedures involving clean-room facilities, high vacuum or complex mask fabrication. Advanced lithographic methods are attracting a lot of interest as a complement to standard lithography aiming to avoid the necessity of the mentioned demanding experimental conditions [12-15]. In this respect electrical [16], chemical $[17,18]$ and mechanical methods [19], block copolymer self-assembly [20], use of templates [21, 22] and laser induced [23] patterning of polymer surfaces are some versatile strategies in order to obtain functional polymer materials. 
In particular, the last few years have witnessed the emergence of a new family of nanofabrication techniques with specific capabilities based in the use of lasers. They constitute attractive alternatives for high-resolution patterning of soft materials affording the sought versatility and reliability. Examples are laser induced periodic surface structuring (LIPSS) [24], laser foaming [25], and other techniques based on laser ablation, as laser interference lithography (LIL) [26], laser induced forward transfer (LIFT) [27], pulsed laser deposition (PLD) [28] and matrix-assisted pulsed laser evaporation (MAPLE) [29]. All these methods can be used to fabricate substrates with a variety of high-precision patterns at different length scales. More recently, specific laser processing techniques, taking advantage of optical trapping or of plasmonic enhancement effects $[30,31]$, have been developed and applied to the nanopatterning of soft polymer materials. In general, laser-based techniques can be applied to many different materials in non-contact and flexible set-ups under a great variety of environments. Moreover, the processing can be tuned both to the materials properties and to the desired surface pattern by controlling the laser parameters like intensity, fluence, wavelength, pulse duration, total photon dose and several other irradiation conditions [32]. When using laser with pulses in the nanosecond (ns) range the material thermal properties determine the outcome of the laser fabrication process. However, for lasers with femtosecond (fs) pulses the laser-material interaction is the key factor governing the surface modification mechanism [33-35]. In this case, the possibility of temporally shaping femtosecond pulses offers new avenues for controlling and tailoring the features of the created structures $[36,37]$.

LIPSS formation was first observed by Birnbaum [38] after ruby-laser irradiation of several semiconductor materials and since then, this type of structures has been generated on a wide variety of materials. LIPSS originate from the interference of the 
incident and reflected/refracted laser light with the scattered light near the interface. The interference between the different waves leads to an inhomogeneous energy input which, together with positive feedback mechanisms, can cause surface instabilities [3941]. LIPSS have been reported on the surface of metals, semiconductors and dielectrics with lasers of different pulse duration from nanosecond to femtosecond, and different wavelengths from the ultraviolet (UV) to the infrared (IR) [42-46]. In the case of polymers, several studies have shown that irradiation by a polarized laser beam induces self-organized ripple structure formation within a narrow fluence range, well below the ablation threshold [39, 44, 45, 47, 48]. As an example Figure 1 illustrates a typical ripple structure on poly(3-hexyl thiophene), $\mathrm{P} 3 \mathrm{HT}$, as observed by atomic force microscopy (AFM) [49].

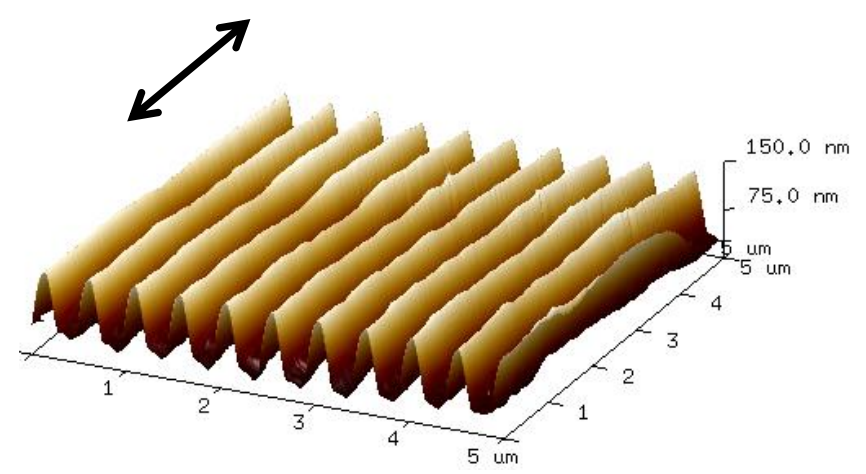

Figure 1. AFM height image of P3HT irradiated at $532 \mathrm{~nm}$ with 4800 pulses at a fluence of $26 \mathrm{~mJ} / \mathrm{cm}^{2}$. The arrow indicates the laser polarization direction. Adapted from reference [49].

The period of the ripples $L$ depends on the laser wavelength and on the angle of incidence of the radiation and their direction is related to the direction of the laser beam polarization [50]. The spacing of the structures can be described by the expression [50]: 


$$
L=\frac{\lambda}{n-\sin (\theta)}
$$

where $\lambda$ is the laser wavelength, $n$ the effective refractive index of the material and $\theta$ the angle of incidence of the laser beam.

For polymers irradiated with nanosecond laser pulses different processes such as thermal and non-thermal scissoring of polymer chains, amorphization of crystalline domains, local surface melting, ablation, photolytic shrinkage, photooxidation and material transport and rearrangement, have been proposed to be involved in ripple formation [24, 39-41, 48, 51]. More recently it has been reported [6, 52] that in order to obtain LIPSS in amorphous polymers, a minimum fluence value is necessary in order to ensure that the surface temperature overcomes the glass transition temperature $\left(T_{\mathrm{g}}\right)$, therefore allowing polymer segmental and chain dynamics. In the case of semicrystalline polymers, the thermal properties are governed not only by the glass transition temperature but also by the melting temperature $\left(T_{\mathrm{m}}\right)$. Thus, the temperature reached at sample surface upon laser irradiation must overcome $T_{\mathrm{m}}$ so that superficial crystallites melt providing enough polymer dynamics [49].

LIPSS generated by femtosecond pulses (fs-LIPSS) in polymers have been mostly observed at the bottom of ablation craters. In this case, together with the classical process of interference between incident and scattered waves, some other mechanisms have been proposed. Particularly, the work of Sipe et al. [53] proposes a first-principle theory which takes into consideration the interaction of an electromagnetic wave with a microscopically rough surface and also includes the possible excitation of surface plasmon polaritons (SPP). In later publications, Young and co-workers, as well as Clark and Emmony, applied this theory to explain the formation of LIPSS in semiconductors [54-56] and metals $[54,56]$. Some other theoretical attempts have been made to 
describe fs-LIPSS based on well-known hydrodynamic models of thin films [57] and on the Sipe-Drude model [43, 58], which considers the laser induced changes in the complex refractive index due to transient generation of quasi-free electrons in the conduction band of the solid. Other authors [59] have proposed different type of mechanisms in which the laser irradiation first originates a highly electrostatically unstable surface followed by emission of high momentum surface ions, which in turn induces an appreciable recoil pressure on the substrate. In this case, ripples would arise from relaxation rather than from wave interference effects. Despite the extensive studies on LIPSS, only few works report about LIPSS on polymers upon irradiation in the femtosecond regime [7, 60-63]. This is in contrast with the wealth of information on laser processing of polymers by nanosecond pulses [6, 24, 64, 65] .

In general, LIPSS can be prepared in both spin-coated $[6,7,52,66,67]$ and in free standing polymer films $[64,68,69]$. This fact makes LIPSS a potential method to obtain large processed surface area and good quality samples. The characteristic surface structures formed in this way, can be used to tailor a great variety of surface properties such as adhesion and friction [51, 70], induced cell alignment [64], liquid crystal alignment [71] and colour generated by superficial gratings [72].

In this paper, we will focus on LIPSS formation on polymers by using either nanosecond or femtosecond pulses. The influence of different irradiation parameters including polarization and angle of incidence will be discussed. Applications of the nanostructured polymer films for cell culture and for Surface Enhanced Raman Scattering (SERS) substrates are described. The possibility of nanostructuring semiconducting polymers with potential applications in organic photovoltaics will also be discussed. 


\section{Laser nanostructuring of polymer films}

\subsection{LIPSS formation in polymers using nanosecond laser pulses}

As a main rule, a high absorption coefficient at the laser wavelength is needed in order to obtain LIPSS upon irradiation with nanosecond pulses. Thus, knowledge of the UVvisible absorption spectra of the polymer is necessary in order to ascertain the possibility of LIPSS formation. In addition, the original samples must present a smooth surface with mean roughness in the range of few nanometers. In principle, LIPSS can be formed on the surface of a great variety of polymers such as polystyrene (PS), poly(ethylene terephthalate) (PET), poly (trimethylene terephthalate) (PTT) and polycarbonate bisphenol A (PBAC) among others [6, 52, 64]. Also naturally-derived polymers such as chitosan and its 50/50 wt. \% blend with the synthetic polymer polyvinylpyrrolidone (PVP) [71, 73] and the poly conjugated polymer poly(3hexylthiophene) (P3HT) have been reported to form LIPSS [49]. Ripple formation appears after laser irradiation at an appropriate wavelength, fluence range, well below the ablation threshold, and with a given number of pulses. The ripple axis is parallel to the polarization vector of the laser beam (see Figure 1). The nanostructured area is restricted to the footprint of the laser beam. Typically, an area of several tens of $\mathrm{mm}^{2}$ can be structured while keeping the sample at a fixed position upon irradiation. Alternatively, sample scanning may be used in case larger nanostructured areas are needed. LIPSS formation depends on the laser fluence. As a typical example, Figure 2 displays AFM height images of areas of PTT irradiated with 1200 pulses at different fluences. For low fluences, no morphological changes are induced on the polymer surface and the roughness remains unchanged. At intermediate fluences (Figure 2b), surface modification starts, as revealed by an increase of roughness. For even higher fluences the structures start to be formed (Figure 2c) in the form of ripples which are 
parallel and well defined in a narrow range of fluences (Figures $2 \mathrm{~d}, \mathrm{e}$ ). Finally, for even higher fluences (Figure 2f) LIPSS become distorted. This trend is general for most polymers although the specific fluence values depend on the particular polymer properties.

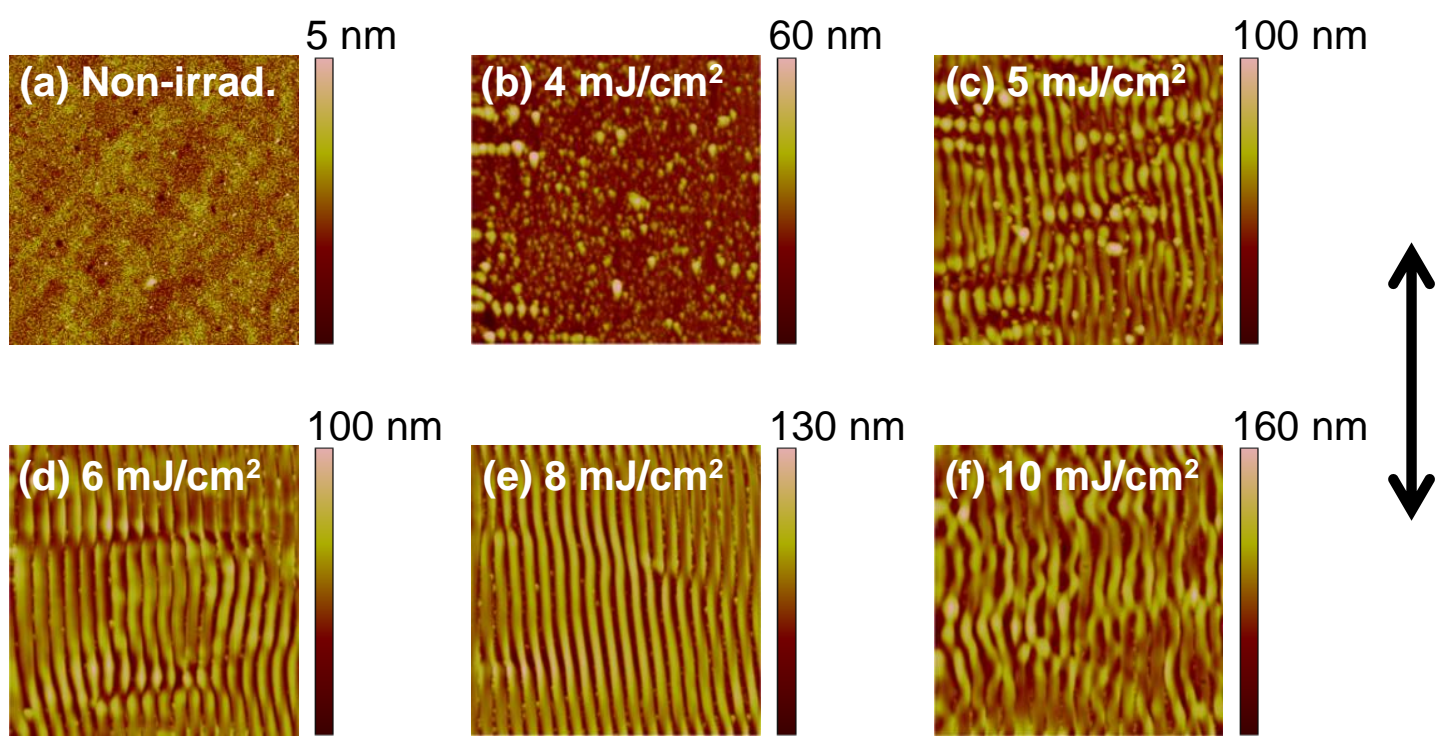

Figure 2. AFM height images $\left(5 \times 5 \mu \mathrm{m}^{2}\right.$ size $)$ of PTT: (a) non-irradiated; irradiated at $266 \mathrm{~nm}$ with 1200 pulses at fluences of: (b) $4 \mathrm{~mJ} / \mathrm{cm}^{2}$, (c) $5 \mathrm{~mJ} / \mathrm{cm}^{2}$, (d) $6 \mathrm{~mJ} / \mathrm{cm}^{2}$, (e) 8 $\mathrm{mJ} / \mathrm{cm}^{2}$, and (f) $10 \mathrm{~mJ} / \mathrm{cm}^{2}$. The arrow indicates the polarization direction. Adapted from reference [6].

For a given fluence, LIPSS formation also depends on the number of pulses as exemplified in Figure 3 for a PET film. As illustrated, a certain amount of pulses, in this case $N>100$, is required in order to obtain well defined LIPSS. 


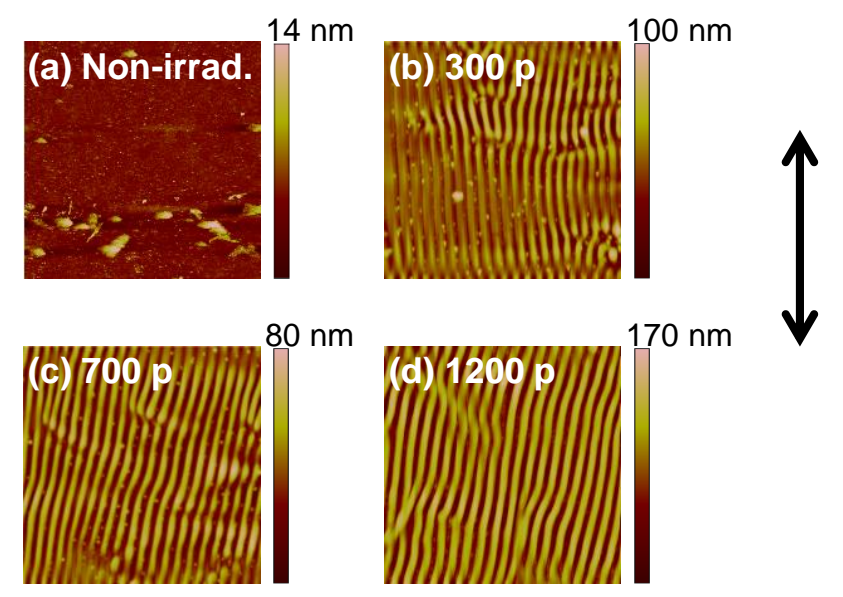

Figure 3. AFM height images $\left(5 \times 5 \mu \mathrm{m}^{2}\right.$ size $)$ of PET: (a) non-irradiated and irradiated at $266 \mathrm{~nm}$ and $6 \mathrm{~mJ} / \mathrm{cm}^{2}$ with (b) 300 pulses, (c) 700 pulses and (d) 1200 pulses. The arrow indicates the polarization direction. Adapted from reference [6].

In general for polymers, the period of the LIPSS increases with increasing fluence until it reaches a plateau for a value of period similar to the irradiating laser wavelength $[6$, 52]. This effect, which is exemplified Figure $4 \mathrm{a}$, is expected according to equation 1 . A similar trend is observed for the dependence of the LIPSS period with the number of pulses (Figure 4b). 

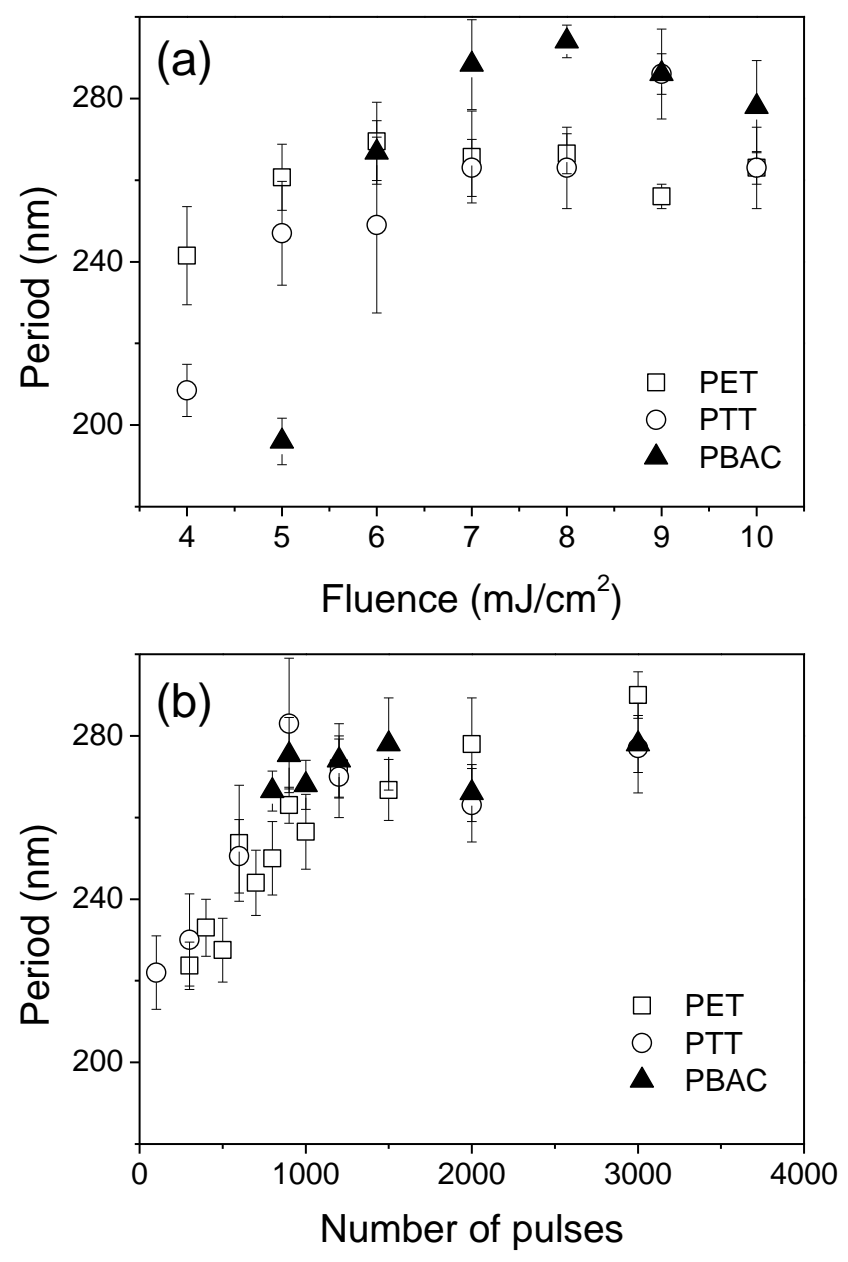

Figure 4. Period of LIPSS formed in PET, PTT and PBAC upon irradiation at $266 \mathrm{~nm}$ (a) as a function of fluence for a fixed amount of pulses $N=600$ and (b) as a function of the number of pulses at $7 \mathrm{~mJ} / \mathrm{cm}^{2}$. Adapted from reference [52].

Regarding the mechanism of LIPSS formation upon irradiation with nanosecond pulses, as mentioned above, it is commonly accepted that interference between the incident laser beam and the surface scattered wave plays a crucial role $[39,50]$. The irradiated surface scatters the incident beam which interferes with the surface wave resulting in a modulated distribution of the energy on the surface. Laser irradiation causes the heating of the upper layer of the polymeric film and the corresponding temperature increase can be estimated as a function of time at different depths from the surface, in a first 
approach, by solving the one-dimensional heat conduction equation $[6,39]$. The results of the calculations show that in order to generate LIPSS, a minimum value of the laser fluence is necessary in order to ensure that the surface temperature overcomes $T_{g}$. This makes possible the devitrification of the surface and therefore it allows polymer segmental and chain dynamics $[6,52]$. The heating above $T_{g}$ is expected to induce an increase of surface roughness caused by capillary waves [73], thus enhancing surface inhomogeneities and facilitating the feedback mechanism involved in LIPSS formation. When comparing polymers with different $T_{g}$ values, like PET, PTT and PBAC, it is observed that the one with higher $T_{g}$, PBAC, requires higher fluences and number of pulses for LIPSS formation [6]. In fact, some authors have proposed that a higher $T_{\mathrm{g}}$ tends to extend the "incubation (induction)" period needed for generation of LIPSS [45]. The estimation of temperature increase upon laser irradiation allows discussing the dependence of ripple period on the fluence in the restricted range at which ripples are formed (Figure 4a). In fact, the increase of fluence leads to the increase of surface temperature which in turn generates a "softer material" with lower superficial viscosity [74]. In general, this allows the formation of wider structures characterized by larger periods.

\subsection{In situ monitoring of LIPSS formation}

The more common way to investigate the morphology of LIPSS is to carry out direct microscopic imaging of the surface by techniques like AFM [38, 41, 61, 63, 64] or scanning electron microscopy (SEM) [36, 46, 47, 63, 65]. The visualization of nanostructures in real space is advantageous since it allows direct assessment of the pattern morphology. However, scattering techniques can be more suitable than those based on microscopy when dealing with fast dynamic processes. Grazing incidence X- 
ray scattering at small (GISAXS) and wide angle (GIWAXS) with synchrotron radiation are appropriate to deal with dynamic processes enabling to obtain reciprocal space structural information evolving even in the millisecond time range [75]. A representative example of the in situ monitoring of LIPSS formation upon repetitive laser irradiation by GISAXS is shown in Figure 5 for a laser repetition rate of $5 \mathrm{~Hz}$. This figure shows a sequence of GISAXS patterns during laser irradiation of a PTT film as a function of both the number of pulses and the corresponding real experimental time. The first pattern, taken after laser irradiation with 25 pulses $(5 \mathrm{~s})$, is still the characteristic one for a spin-coated film in absence of relevant structure in the angular range investigated. The GISAXS patterns start exhibiting clear vertical diffraction maxima after irradiation with 200 pulses (40 s). Scattering maxima out of the meridian $(\omega \neq 0)$ are clearly visible in the range of LIPSS formation. The scattering patterns have shown to be characteristic of a one-dimensional paracrystalline lattice formed by the ripples $[52,76,77]$. For higher number of pulses the vertical diffraction maxima increase both in number and in intensity. The period of the LIPSS can be derived from the lateral position of the first maxima [48]. The AFM images at the bottom of the image illustrate the initial and the final state of the polymer film. In situ monitoring of LIPSS formation have shown that the dynamics of nanostructure formation is dependent on the laser repetition rate and this can be related to the feedback mechanism [6]. For $10 \mathrm{~Hz}$, a much lower number of pulses $(\approx 30)$ is needed for the appearance of LIPSS in comparison to the number of pulses $(\geq 100)$ required at lower repetition rates. The process is more efficient for a shorter time separation between successive pulses. 


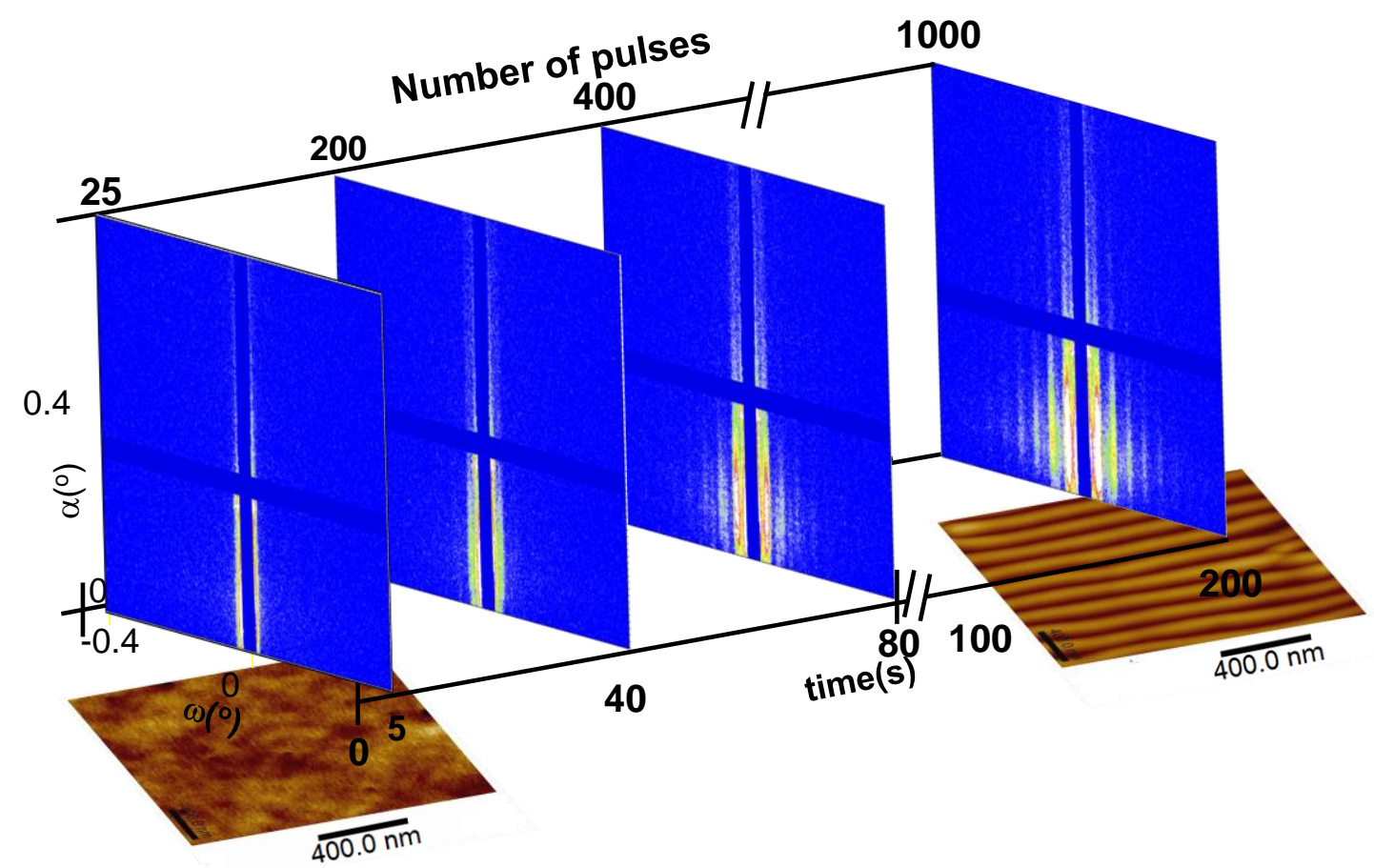

Figure 5. Sequence of GISAXS patterns acquired in situ as a function of both time and number of pulses for a PTT film irradiated at $266 \mathrm{~nm}$ with a laser repetition rate of $5 \mathrm{~Hz}$ and with a fluence of $7 \mathrm{~mJ} / \mathrm{cm}^{2}$. The bottom AFM images $\left(2 \times 2 \mu \mathrm{m}^{2}\right)$ illustrate the initial and the final state of the polymer film. Adapted from reference [76].

\subsection{Different approaches for the formation of alternative periodic structures}

\subsubsection{LIPSS with different periods: variation of incidence angle}

The period of LIPSS can be modified by changing the laser wavelength but also by varying the angle of incidence of the laser with respect to the surface according to equation 1 [50]. By increasing the angle of incidence the period of the structures increases. Figure 6a-6c shows LIPSS with periods of around 250, 320 and $490 \mathrm{~nm}$ that were measured for angles of incidence of $0^{\circ}, 22^{\circ}$ and $45^{\circ}$, respectively, upon irradiation at a wavelength of $266 \mathrm{~nm}$ [67]. It is important to mention that the amplitude of the structures obtained also increases for larger periods, from 50 to $120 \mathrm{~nm}$. 


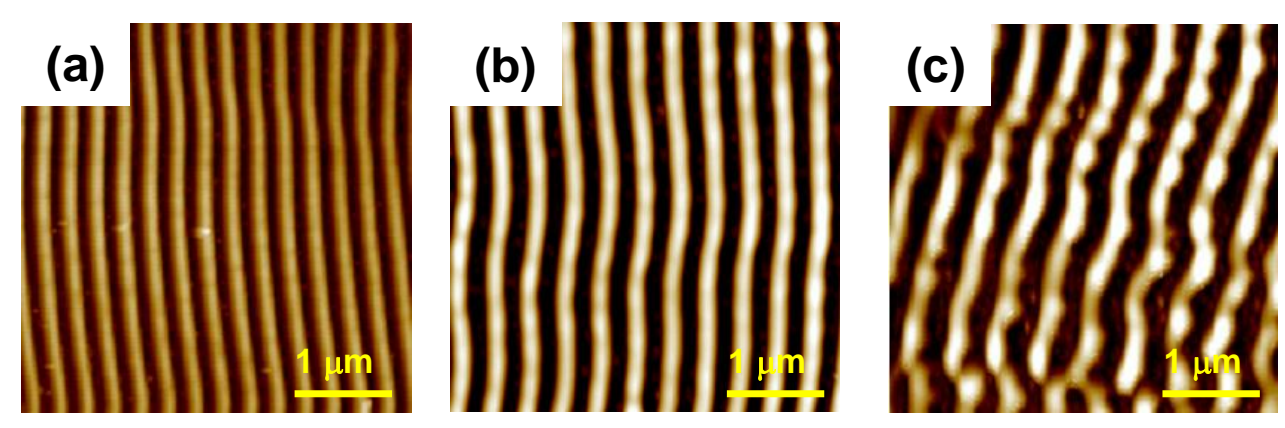

Figure 6. AFM height images $\left(4 \mathrm{x} 4 \mu \mathrm{m}^{2}\right)$ of a PET film irradiated with a linearly polarized beam at $266 \mathrm{~nm}$ using 1200 pulses and $7 \mathrm{~mJ} / \mathrm{cm}^{2}$ at: (a) normal incidence, (b) $22^{\circ}$ and (c) $45^{\circ}$. (Adapted from reference [67]).

\subsubsection{LIPSS with different shapes: variation of polarization.}

It is worth mentioning that certain control over the morphology of the superficial structures can be achieved by changing the polarization of the laser beam. Either linear or circular polarization can be obtained by inserting a quarter-wave plate in the laser beam path. Figure $7 \mathrm{a}-7 \mathrm{c}$ shows nanostructures of different shape obtained by changing the state of polarization of the beam. Irradiation with a linearly polarized beam induces standard LIPSS formation parallel to the polarization vector of the beam (Figure 7a). By rotating the quarter-wave plate, the polarization is changed to elliptic and, as seen in Figure $7 b$, the ripples appear aligned to the larger axis of the polarization ellipse. A very different surface pattern appears for circular polarization (Figure 7c). In this case the sample is covered by uniformly distributed nanodots with a diameter of around $260 \mathrm{~nm}$, similar to the laser wavelength. This change of morphology must be related to the fact that the amplitude of the electric vector for circularly polarized light is constant and rotates around the propagation direction in such a way that the irradiated and subsequently heated and rearranged material aligns to the successive polarization directions and gives rise to a uniformly distributed pattern. 

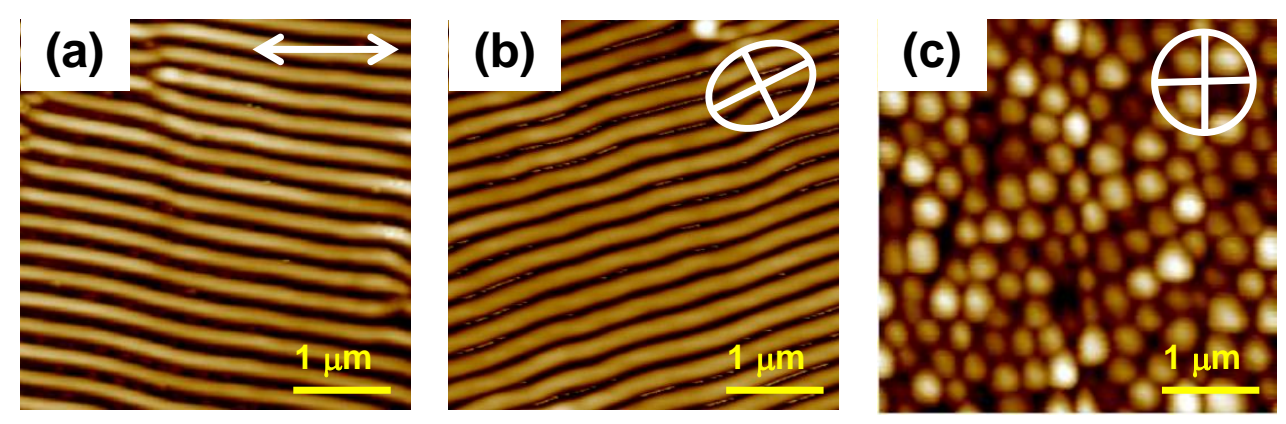

Figure 7. PET film irradiated at $266 \mathrm{~nm}$ using 1200 pulses and $7 \mathrm{~mJ} / \mathrm{cm}^{2}$ with polarization (a) linear; (b) elliptical, and (c) circular. Reproduced from Ref. [67] with permission.

\subsection{LIPSS formation in polymers using femtosecond laser pulses}

As mentioned above, significant absorption at the laser wavelength is required in order to obtain LIPSS with nanosecond pulses. However, it has been reported that LIPSS can be fabricated upon irradiation with femtosecond pulses at a wavelength at which the material has a low linear absorption coefficient. Figure 8 illustrates the case for PET films irradiated at the fundamental wavelength of a Ti:Sapphire laser (795 nm) [7, 66]. Upon irradiation with femtosecond pulses, due to the large laser intensities involved, multiphoton absorption and ionization processes mediate the coupling of laser light with the outer layer of the polymer film. This makes possible the formation of LIPSS at this long wavelength at which the absorbance of the polymers is very small. In this case the period and height of the LIPSS structures tend to increase with both fluence and number of pulses towards the value of the laser wavelength [66]. 

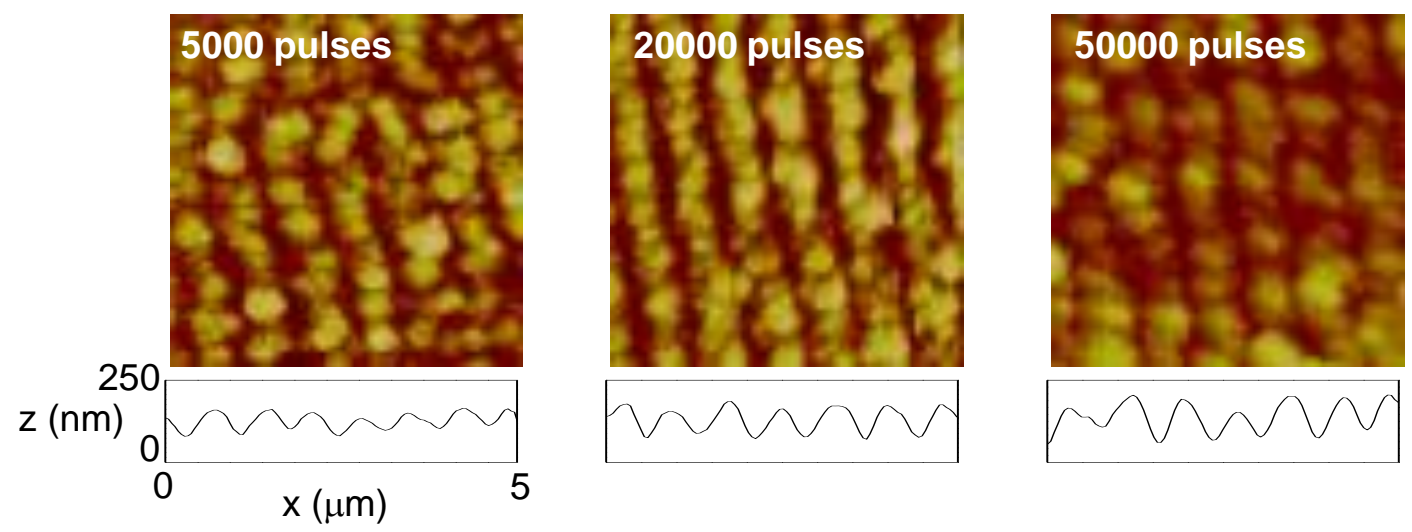

Figure 8 . AFM height images $\left(5 \times 5 \mathrm{~m}^{2}\right)$ of PET irradiated at $795 \mathrm{~nm}$ with pulses of $120 \mathrm{fs}$ and different pulses, as indicated, at the fluence of $37 \mathrm{~mJ} / \mathrm{cm}^{2}$. The height profile along a $5 \mu \mathrm{m}$ line perpendicular to the ripples is shown below every image. Adapted from reference [66].

\subsection{Physico-chemical modifications upon LIPSS formation}

Besides the topographical changes previously described, additional physico-chemical modifications can be induced upon nanostructuring of the polymer surfaces. The extent of chemical changes can be inspected for example by micro-Raman spectroscopy [67]. Figure 9 shows Raman spectra corresponding to a film of PET before and after irradiation using different laser conditions. Changes upon irradiation of the band at 1615 $\mathrm{cm}^{-1}$, which is assigned to the ring stretching, can be detected. As shown, this band shifts by $\sim 2 \mathrm{~cm}^{-1}$ to a lower wavenumber, and broadens somewhat for samples irradiated at $266 \mathrm{~nm}$ with circular polarization and with linear polarization at $45^{\circ}$. These changes suggest a rearrangement of the polymer chains after irradiation. Figure 9 also shows the stretching vibration of the $\mathrm{C}=\mathrm{O}$ bond (band at $1726 \mathrm{~cm}^{-1}$ ). Upon irradiation it reveals a shoulder at lower wavenumbers which could be assigned to carboxylic acid group formation. By comparing the total integrated intensity in the $1650-1800 \mathrm{~cm}^{-1}$ 
spectral region it is observed that the total $\mathrm{C}=\mathrm{O}$ content increases. These results suggest that irradiated samples contain a higher amount of oxygen atoms due to the appearance of new carboxylic and carbonyl groups, which can be ascribed to surface oxidation. Contact angle (CA) measurements in this case provide information on the strength of non-covalent forces between the liquid and the substrate surface. For irradiated samples, the CA of water (also shown in Figure 9) decreases, indicating that the surface displays a more hydrophilic character after irradiation. Changes occur at a higher extension for the substrates irradiated with circular polarization.

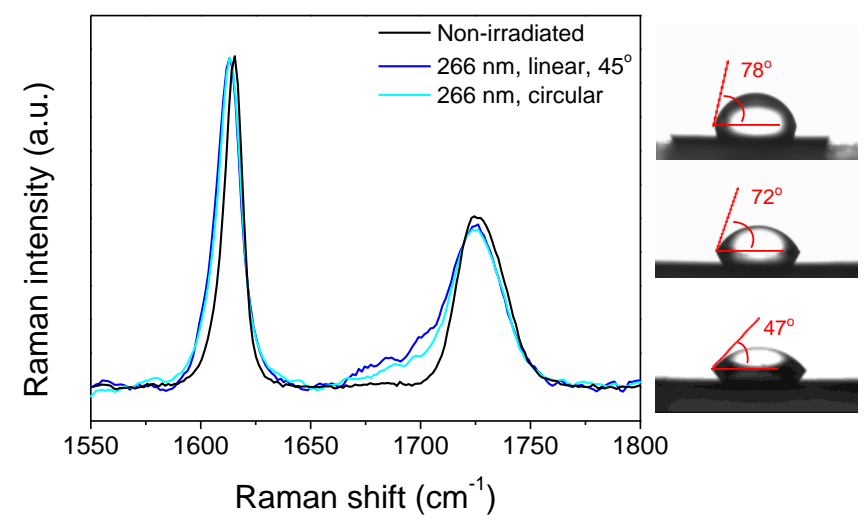

Figure 9. Raman spectra of a PET film before and after irradiation at $266 \mathrm{~nm}, 1200$ pulses and $7 \mathrm{~mJ} / \mathrm{cm}^{2}$ with linear polarization at an angle of incidence of $45^{\circ}$ and with circular polarization. For comparison, spectra have been baseline corrected and the intensities normalized to that of the band at $1615 \mathrm{~cm}^{-1}$. To the right, images of a water drop on the different surfaces are shown: non-irradiated (upper), irradiated with linear polarization at an angle of incidence of $45^{\circ}$ (center) and irradiated with circular polarization (bottom). Adapted from reference [67].

\section{Applications of polymer LIPSS}


Once we have shown that it is possible to generate LIPSS with different sizes and shapes we will describe how these substrates can be appropriate for different applications. In particular, in the following paragraphs we will show examples of the use of polymer LIPSS for cell culture, for SERS substrates, and of the nanostructuring of semiconducting polymers of interest in photovoltaics.

\subsection{Polymer LIPSS for cell culture}

LIPSS generated on PET films with different sizes and shapes have been used for mesenchymal cell culture tests as illustrated in Figure 10. By comparing the culture on non-irradiated samples (Fig. 10a) to that on a polymer with LIPSS, it can be inferred that nanostructures promote in all cases cell adhesion and growth [67]. It is important to note that samples irradiated at 266 with circularly polarized laser light (Figure 10e) exhibit a larger increase of oxygen groups as mentioned above, and a higher value of surface free energy as determined by CA measurements, indicating that these characteristics can be relevant for cell culture [67].
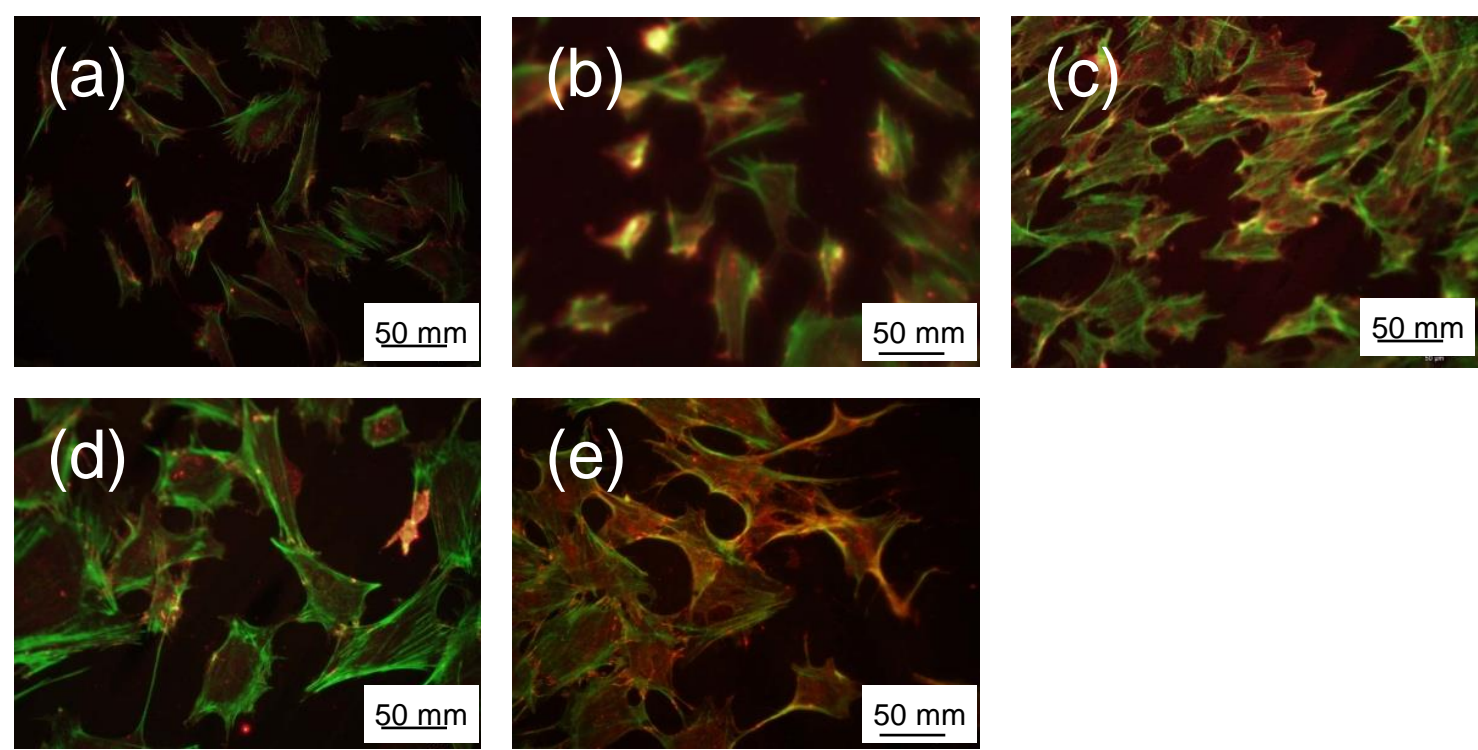
Figure 10. Microscopy images of cell cultured PET films: (a) non-irradiated, (b) irradiated at $193 \mathrm{~nm}$, (c) irradiated at $266 \mathrm{~nm}$, linearly polarized laser at normal incidence, (d) irradiated at $266 \mathrm{~nm}$, linearly polarized, incidence at $45^{\circ}$, (e) irradiated at $266 \mathrm{~nm}$, circularly polarized. Adapted from reference [67].

As another example, it has been reported that LIPSS generated on PS films [64] with different periodicities have been used for culturing human embryonic kidney (HEK293) cells, Chinese hamster ovary (CHO-K1) cells and skeletal myoblasts. It was shown that adhesion and proliferation of cells is enhanced for the polymer LIPSS in comparison to the unmodified PS. Furthermore, the cells align along the direction of the ripples, although the alignment is cell-type dependent and occurs only when the periodicity of the nanostructures is above a critical value. Thus, while HEK-293 and CHO-K1 cells align along the direction of the ripples for periods equal or above 340 $\mathrm{nm}$, rat skeletal myoblast align on the ripples with periodicities equal or above $430 \mathrm{~nm}$ and human myoblasts only align for periodicities equal or above $270 \mathrm{~nm}$.

\subsection{Polymer LIPSS for Surface Enhanced Raman Spectroscopy (SERS)}

\section{substrates}

Polymer LIPSS can be used as Surface Enhanced Raman Scattering (SERS) sensors $[78,79]$. To that purpose LIPSS nanostructured polymer films were coated with a gold layer by pulsed laser deposition (PLD). The morphology of the gold coated polymer samples retained the initial periodic relief after PLD coating as can be observed in figure 11. Raman spectra were acquired on drops of aqueous solutions of the model analyte benzenethiol (BT) in different concentrations, poured onto the gold coated 
polymer substrates and dried in air. The enhancement factor of the Raman signal for the gold-coated substrates is of eight orders of magnitude and an additional enhancement is achieved by the presence of the periodic structures. Figure 11 compares the spectra obtained for a BT concentration of $10^{-5} \mathrm{M}$ on a substrate consisting of unstructured and nanostructured PTT coated with gold both by PLD with 24000 laser pulses of $213 \mathrm{~nm}$ and $2 \mathrm{~J} / \mathrm{cm}^{2}$ which resulted in a gold layer of ca. $10 \mathrm{~nm}$ thickness. The bands observed are readily assigned to the analyte. The observed enhancement effect of around a factor of 10 is due to the presence of the periodic nanostructures. Thus, in comparison with pure gold substrates the above example indicates that a very low amount of gold applied on the polymer LIPSS is enough to observe a substantial enhancement of the Raman signal.
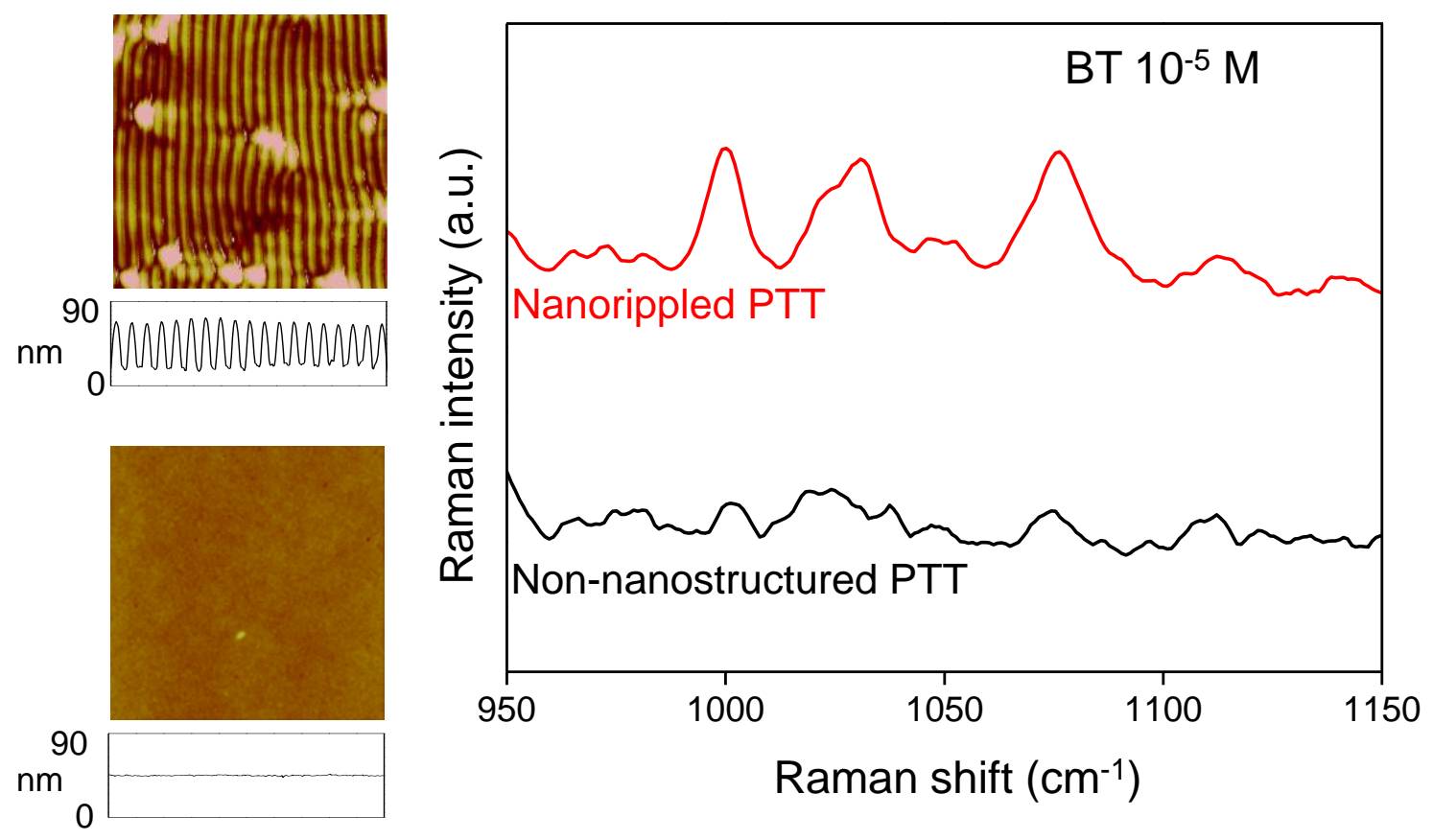

Figure 11. (Left) AFM height images $\left(5 \times 5 \mu \mathrm{m}^{2}\right)$ of non-irradiated PTT and nanostructured PTT both gold coated by PLD. The height profile along a $5 \mu \mathrm{m}$ line perpendicular to the ripples is shown below every image. (Right) Raman spectra of BT 
at a concentration of $10^{-5} \mathrm{M}$ on gold coated non-nanostructured and nanostructured PTT substrates. Adapted from reference [78].

\subsection{LIPSS in semiconducting polymers}

The semiconducting semicrystalline polymer $\mathrm{P} 3 \mathrm{HT}$ has been widely studied as the active layer in organic field-effect transistors (OFET) [80-82] and organic solar cells [83-85]. Besides common patterning methods, it is also possible to fabricate LIPSS on films of this material (Figure 1) [49, 86]. Interestingly enough, LIPSS in P3HT generate regions of different electrical conductivity as illustrated in Figure 12. Here, conductive force microscopy, C-AFM, can be used to characterize the electrical properties at the nanoscale of P3HT films with LIPSS generated at $532 \mathrm{~nm}$. Figure 12 shows the C-AFM current images, acquired in contact mode, of a P3HT thin film sample before and after irradiation with a fluence of $26 \mathrm{~mJ} / \mathrm{cm}^{2}$ and 4800 pulses, by applying a constant voltage of $-5 \mathrm{~V}$ on the conducting substrate. The current image of the sample before irradiation (Figure 12a) shows that the film is conductive in the whole area. The image displaying the current map of the irradiated polymer film (Figure 12b) shows trenches with a similar conductivity to that of the initial film separated by nonconductive ridges.

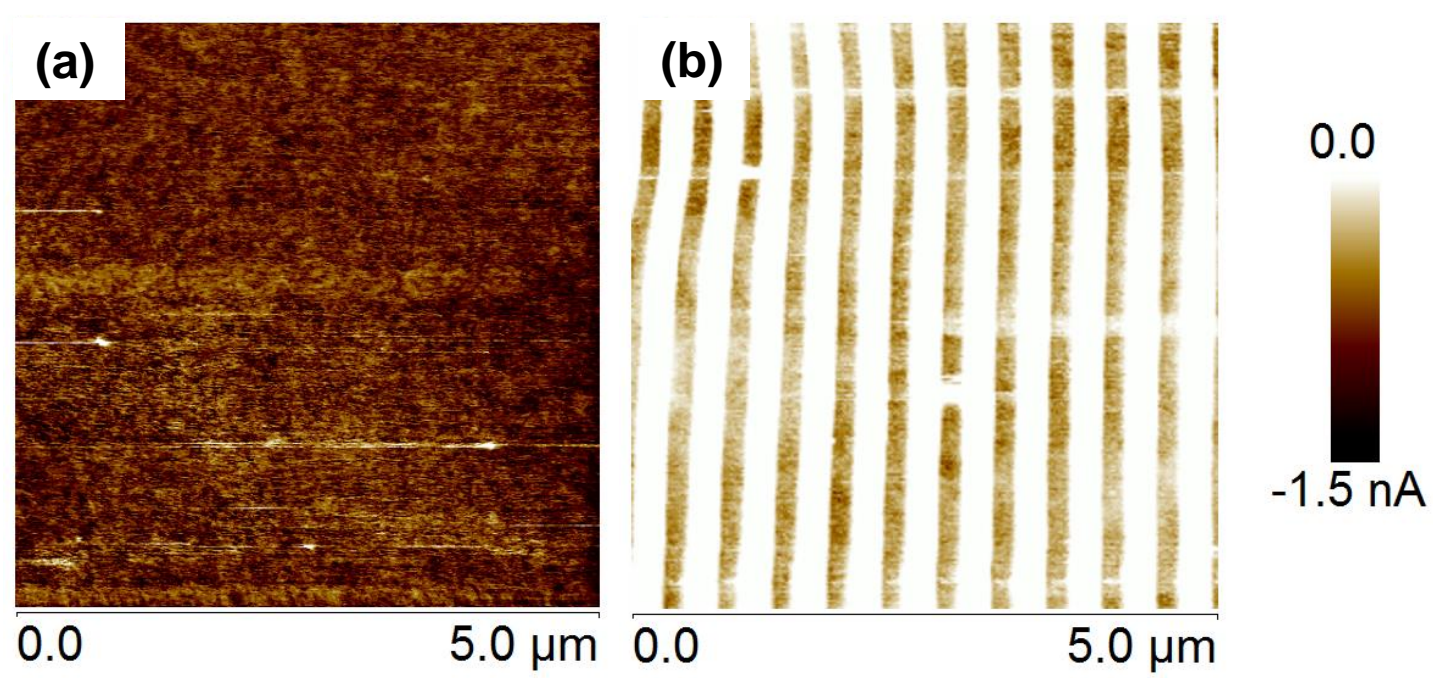


Figure 12. C-AFM current images of a P3HT thin film measured at a constant bias of -5 V. a) Non-irradiated film, b) film with LIPSS fabricated at $532 \mathrm{~nm}, 26 \mathrm{~mJ} / \mathrm{cm}^{2}$ and 4800 pulses. Adapted from reference [49].

Spectroscopic investigation has revealed the absence of significant chemical changes in the P3HT films after LIPSS formation. Raman and GIWAXS results suggest that laser irradiation of $\mathrm{P} 3 \mathrm{HT}$ at the initial stage melts the polymer surface and generates ridges with lower molecular order than that of the original thin film [49]. The ridges are over a continuous P3HT thin film whose initial orientation and crystallinity seems to be unaffected. As a consequence, electrical conduction in the ridges is smaller than that in the trenches.

\section{Conclusions and outlook}

Laser-induced Periodic Surface Structures with periods similar to the laser wavelength, and parallel to the laser polarization direction, are observed in films of strongly absorbing polymers in a narrow range of fluences. LIPSS formation takes place by heating the film surface above the characteristic glass transition temperature of amorphous polymers or above the melting temperature in the case of semicrystalline ones. Grazing incidence X-Ray Scattering techniques are introduced for the study of LIPSS to assess morphology order over large sample areas. In addition, GISAXS can be used for in in situ monitoring of LIPSS formation and allows the study of the role of the laser repetition rate in the development of the superficial nanostructures. The results obtained support the presence of a feedback effect for LIPSS formation in polymer films. 
Different types of structures can be obtained by changing the laser parameters. Thus, while the period of the ripples is close to the laser wavelength for irradiation at normal incidence, it increases for irradiation at different angles. Additionally, the use of circularly polarized light gives rise to circular structures with diameters close to the laser wavelength.

In order to overcome the limitation of this method concerning the necessity of a high linear absorption coefficient at the irradiation wavelength, femtosecond lasers may be used, which allows tuning and controlling the morphology and size of periodic structures on the surface of polymer films.

Different applications of polymer substrates with LIPSS are proposed and demonstrated. The first application refers to cell culture. A higher cell proliferation rate has been observed for samples in which larger surface modifications were induced by laser irradiation in terms of surface free energy. Additionally, nanostructured polymer substrates are proposed for cell alignment. A second application concerns the use of polymer LIPSS as SERS substrates. Coating with gold by pulsed laser deposition has been shown to preserve the relief of the nanostructured polymer films and to ensure good adhesion. Gold coating of the films induces an enhancement of 8 orders of magnitude, while an additional enhancement of one order of magnitude arises by the presence of LIPSS. This approach allows reducing the quantity of precious metal needed to prepare SERS substrates. Finally, it has been shown that LIPSS can be prepared on films of a semiconducting polymer such as P3HT, preserving its chemical integrity and producing nanometric regions different electrical conductivity.

The LIPSS technique has demonstrated to be advantageous due to its simplicity and versatility. Structures in the range $170-800 \mathrm{~nm}$ can be obtained by selecting adequate irradiation parameters. It is important to mention that this laser treatment can easily be 
extended to large surfaces. The characteristic surface structures formed in this way are being used to tailor a great variety of surface properties and as shown display high potential for applications in different fields.

\section{Acknowledgements}

The authors gratefully acknowledge financial support from the MINECO (CTQ 201343086-P, MAT2012-33517 and MAT2011-23455). E.R. also thanks MINECO for a Ramón y Cajal contract (RYC-2011-08069).

\section{References}

[1] Huber G, Mantz H, Spolenak R, Mecke K, Jacobs K, Gorb SN, et al. Evidence for capillarity contributions to gecko adhesion from single spatula nanomechanical measurements. Proc Natl Acad Sci U S A. 2005;102(45):16293-16296.

[2] Li XM, Reinhoudt D, Crego-Calama M. What do we need for a superhydrophobic surface? A review on the recent progress in the preparation of superhydrophobic surfaces. Chemical Society Reviews. 2007;36(8):1350-1368.

[3] Rebollar E, Bounos G, Oujja M, Georgiou S, Castillejo M. Effect of Molecular Weight on the Morphological Modifications Induced by UV Laser Ablation of Doped Polymers. J Phys Chem B. 2006;110(33):16452-16458.

[4] Fernandez JG, Mills CA, Samitier J. Complex Microstructured 3D Surfaces Using Chitosan Biopolymer. Small. 2009;5(5):614-620.

[5] Ranella A, Barberoglou M, Bakogianni S, Fotakis C, Stratakis E. Tuning cell adhesion by controlling the roughness and wettability of 3D micro/nano silicon structures. Acta Biomaterialia. 2010;6(7):2711-2720.

[6] Rebollar E, Pérez S, Hernández JJ, Martín-Fabiani I, Rueda DR, Ezquerra TA, et al. Assessment and Formation Mechanism of Laser-Induced Periodic Surface Structures on Polymer Spin-Coated Films in Real and Reciprocal Space. Langmuir. 2011;27(9):5596-5606. [7] Rebollar E, R. Vazquez de Aldana J, Perez-Hernandez JA, Ezquerra TA, Moreno P, Castillejo M. Ultraviolet and infrared femtosecond laser induced periodic surface structures on thin polymer films. Appl Phys Lett. 2012;100(4):041106-041104.

[8] Schift H. Nanoimprint lithography: An old story in modern times? A review. J Va Sci Technol B. 2008;26(2):458-480.

[9] Guo LJ. Nanoimprint lithography: Methods and material requirements. Adv Mater. 2007;19(4):495-513.

[10] Qin D, Xia YN, Whitesides GM. Soft lithography for micro- and nanoscale patterning. Nat Protoc. 2010;5(3):491-502.

[11] Ho CC, Chen PY, Lin KH, Juan WT, Lee WL. Fabrication of Monolayer of Polymer/Nanospheres Hybrid at a Water-Air Interface. ACS Appl Mater Interfaces. 2011;3(2):204-208. 
[12] Schift H. NaPANIL-Library of Processes. Berlin: NaPANIL consortium; 2012.

[13] Müller-Buschbaum P, Bauer E, Maurer E, Schlögl K, Roth SV, Gehrke R. Route to create large-area ordered polymeric nanochannel arrays. Appl Phys Lett. 2006;88(8):083114.

[14] Müller-Buschbaum P, Bauer E, Pfister S, Roth SV, Burghammer M, Riekel C, et al. Creation of multi-scale stripe-like patterns in thin polymer blend films. Europhysics Letters. 2006;73(1):35-41.

[15] Zhang P, Huang H-y, Chen Y, Yu S, Krywka C, Vayalil S, et al. Preparation of long-range ordered nanostructures in semicrystalline diblock copolymer thin films using micromolding.

Chin J Polym Sci. 2014;32(9):1188-1198.

[16] Schaffer E, Thurn-Albrecht T, Russell TP, Steiner U. Electrically induced structure formation and pattern transfer. Nature. 2000;403(6772):874-877.

[17] Dorrer C, Ruhe J. Mimicking the stenocara beetle-dewetting of drops from a patterned superhydrophobic surface. Langmuir. 2008;24(12):6154-6158.

[18] Wohlfart E, Fernandez-Blazquez JP, Knoche E, Bello A, Perez E, Arzt E, et al. Nanofibrillar Patterns by Plasma Etching: The Influence of Polymer Crystallinity and Orientation in Surface Morphology. Macromolecules. 2010;43(23):9908-9917.

[19] Gong JL, Lipomi DJ, Deng JD, Nie ZH, Chen X, Randall NX, et al. Micro- and Nanopatterning of Inorganic and Polymeric Substrates by Indentation Lithography. Nano Lett. 2010;10(7):2702-2708.

[20] Ruiz R, Kang HM, Detcheverry FA, Dobisz E, Kercher DS, Albrecht TR, et al. Density multiplication and improved lithography by directed block copolymer assembly. Science. 2008;321(5891):936-939.

[21] Martin J, Mijangos C, Sanz A, Ezquerra TA, Nogales A. Segmental Dynamics of Semicrystalline Poly(vinylidene fluoride) Nanorods. Macromolecules. 2009;42(14):5395-5401. [22] Garcia-Gutierrez MC, Linares A, Hernandez JJ, Rueda DR, Ezquerra TA, Poza P, et al. Confinement-Induced One-Dimensional Ferroelectric Polymer Arrays. Nano Lett. 2010;10(4):1472-1476.

[23] Balan L, Turck C, Soppera O, Vidal L, Lougnot DJ. Holographic Recording with Polymer Nanocomposites Containing Silver Nanoparticles Photogenerated in Situ by the Interference Pattern. Chem Mater. 2009;21(24):5711-5718.

[24] Bolle M, Lazare S, Le Blanc M, Wilmes A. Submicron periodic structures produced on polymer surfaces with polarized excimer laser ultraviolet radiation. Appl Phys Lett.

1992;60(6):674-676.

[25] Lazare S, Tokarev V, Sionkowska A, Wiśniewski M. Surface foaming of collagen, chitosan and other biopolymer films by $\mathrm{KrF}$ excimer laser ablation in the photomechanical regime. Appl Phys A. 2005;81(3):465-470.

[26] Yu F, Li P, Shen H, Mathur S, Lehr C-M, Bakowsky U, et al. Laser interference lithography as a new and efficient technique for micropatterning of biopolymer surface.

Biomaterials. 2005;26(15):2307-2312.

[27] Thomas B, Alloncle AP, Delaporte P, Sentis M, Sanaur S, Barret M, et al. Experimental investigations of laser-induced forward transfer process of organic thin films. Appl Surf Sci. 2007;254(4):1206-1210.

[28] Sirajuddin M, Reddy PJ. Pyroelectric behaviour of laser-evaporated poly(vinyl fluoride) films. Thin Solid Films. 1985;124(2):149-154.

[29] Pique A, McGill RCRA, Chrisey DB, Callahan J, Mlsna TE. Matrix assisted pulsed laser evaporation (MAPLE) of polymeric materials: Methodology and mechanistic studies. Materials Research Society Symposium - Proceedings1998. p. 375-383.

[30] Tsai YC, Leitz KH, Fardel R, Otto A, Schmidt M, Arnold CB. Parallel optical trap assisted nanopatterning on rough surfaces. Nanotechnology. 2012;23(16):165304.

[31] Vazquez-Mena O, Sannomiya T, Tosun M, Villanueva LG, Savu V, Voros J, et al. High-

Resolution Resistless Nanopatterning on Polymer and Flexible Substrates for Plasmonic

Biosensing Using Stencil Masks. ACS Nano. 2012;6(6):5474-5481.

[32] Bäuerle D. Laser processing and chemistry. Berlin: Springer-Verlag; 2011. 
[33] Rebollar E, Cordero D, Martins A, Chiussi S, Reis RL, Neves NM, et al. Improvement of electrospun polymer fiber meshes pore size by femtosecond laser irradiation. Appl Surf Sci. 2011;257(9):4091-4095.

[34] Chichkov BN, Momma C, Nolte S, von Alvensleben F, Tünnermann A. Femtosecond, picosecond and nanosecond laser ablation of solids. Appl Phys A. 1996;63(2):109-115.

[35] Liu X, Du D, Mourou G. Laser ablation and micromachining with ultrashort laser pulses. Quantum Electronics, IEEE Journal of. 1997;33(10):1706-1716.

[36] Englert L, Wollenhaupt M, Haag L, Sarpe-Tudoran C, Rethfeld B, Baumert T. Material processing of dielectrics with temporally asymmetric shaped femtosecond laser pulses on the nanometer scale. Appl Phys A. 2008;92(4):749-753.

[37] Rebollar E, Mildner J, Götte N, Otto D, Sarpe C, Köhler J, et al. Microstructuring of soft organic matter by temporally shaped femtosecond laser pulses. Appl Surf Sci. 2014;302(0):231235.

[38] Birnbaum M. Semiconductor Surface Damage Produced by Ruby Lasers. J Appl Phys. 1965;36(11):3688-3689.

[39] Csete M, Bor Z. Laser-induced periodic surface structure formation on polyethyleneterephthalate. Appl Surf Sci. 1998;133(1-2):5-16.

[40] Bolle M, Lazare S. Characterization of submicrometer periodic structures produced on polymer surfaces with low-fluence ultraviolet laser radiation. J Appl Phys. 1993;73(7):35163524.

[41] Prendergast U, Kudzma S, Sherlock R, O'Connell C, Glynn T. TEM investigation of laserinduced periodic surface structures on polymer surfaces. Photon Processing in Microelectronics and Photonics VI. 1 ed. San Jose, CA, USA: Proc. SPIE; 2007. p. 64581V-64510.

[42] Reif J, Varlamova O, Ratzke M, Schade M, Leipner H, Arguirov T. Multipulse feedback in self-organized ripples formation upon femtosecond laser ablation from silicon. Appl Phys A. 2010;101(2):361-365.

[43] Bonse J, Kruger J. Pulse number dependence of laser-induced periodic surface structures for femtosecond laser irradiation of silicon. J Appl Phys. 2010;108(3):034903-034905.

[44] Csete M, Hild S, Plettl A, Ziemann P, Bor Z, Marti O. The role of original surface roughness in laser-induced periodic surface structure formation process on poly-carbonate films. Thin Solid Films. 2004;453-454:114-120.

[45] Li M, Lu QH, Yin J, Sui Y, Li G, Qian Y, et al. Periodic microstructure induced by $532 \mathrm{~nm}$ polarized laser illumination on poly(urethane-imide) film: orientation of the azobenzene chromophore. Appl Surf Sci. 2002;193(1-4):46-51.

[46] Sanz M, Rebollar E, Ganeev RA, Castillejo M. Nanosecond laser-induced periodic surface structures on wide band-gap semiconductors. Appl Surf Sci. 2013;278(0):325-329.

[47] Lazare S, Benet P. Surface amorphization of Mylar[sup (R)] films with the excimer laser radiation above and below ablation threshold: Ellipsometric measurements. J Appl Phys. 1993;74(8):4953-4957.

[48] Csete M, Eberle R, Pietralla M, Marti O, Bor Z. Attenuated total reflection measurements on poly-carbonate surfaces structured by laser illumination. Appl Surf Sci. 2003;208-209:474480.

[49] Rodríguez-Rodríguez Á, Rebollar E, Soccio M, Ezquerra TA, Rueda DR, Garcia-Ramos JV, et al. Laser-Induced Periodic Surface Structures on Conjugated Polymers: Poly(3hexylthiophene). Macromolecules. 2015.

[50] Bäuerle D. Laser processing and chemistry. Berlin: Springer-Verlag; 2000.

[51] Bolle M, Lazare S. Submicron periodic structures produced on polymer surfaces with polarized excimer laser ultraviolet radiation. Appl Surf Sci. 1993;65-66:349-354.

[52] Martín-Fabiani I, Rebollar E, Pérez S, Rueda DR, García-Gutiérrez MC, Szymczyk A, et al. Laser-Induced Periodic Surface Structures Nanofabricated on Poly(trimethylene terephthalate) Spin-Coated Films. Langmuir. 2012;28(20):7938-7945.

[53] Sipe JE, Young JF, Preston JS, van Driel HM. Laser-induced periodic surface structure. I. Theory. Phys Rev B. 1983;27(2):1141. 
[54] Young JF, Preston JS, van Driel HM, Sipe JE. Laser-induced periodic surface structure. II. Experiments on Ge, Si, Al, and brass. Phys Rev B. 1983;27(2):1155.

[55] Young JF, Sipe JE, van Driel HM. Laser-induced periodic surface structure. III. Fluence regimes, the role of feedback, and details of the induced topography in germanium. Phys Rev B. 1984;30(4):2001.

[56] Clark SE, Emmony DC. Ultraviolet-laser-induced periodic surface structures. Phys Rev B. 1989;40(4):2031-2041.

[57] Bestehorn M, Pototsky A, Thiele U. 3D Large scale Marangoni convection in liquid films. The European Physical Journal B - Condensed Matter and Complex Systems. 2003;33(4):457467.

[58] Dufft D, Rosenfeld A, K. Das S, Grunwald R, Bonse J. Femtosecond laser-induced periodic surface structures revisited: A comparative study on ZnO. J Appl Phys. 2009;105(3):034908034909.

[59] Costache F, Henyk M, Reif J. Modification of dielectric surfaces with ultra-short laser pulses. Appl Surf Sci. 2002;186(1-4):352-357.

[60] Baudach S, Bonse J, Kautek W. Ablation experiments on polyimide with femtosecond laser pulses. Applied Physics A: Materials Science and Processing. 1999;69(7):S395-S398.

[61] Forster M, Kautek W, Faure N, Audouard E, Stoian R. Periodic nanoscale structures on polyimide surfaces generated by temporally tailored femtosecond laser pulses. Phys Chem Chem Phys. 2011;13(9):4155-4158.

[62] Krüger J, Kautek W. Ultrashort Pulse Laser Interaction with Dielectrics and Polymers. In: Lippert TK, editor. Polymers and Light: Springer Berlin / Heidelberg; 2004. p. 247-290.

[63] Baudach S, Krüger J, Kautek W. Femtosecond laser processing of soft materials. Rev Laser Eng 2001;29:705-709.

[64] Rebollar E, Frischauf I, Olbrich M, Peterbauer T, Hering S, Preiner J, et al. Proliferation of aligned mammalian cells on laser-nanostructured polystyrene. Biomaterials. 2008;29(12):17961806.

[65] Dyer PE, Farley RJ. Periodic surface structures in the excimer laser ablative etching of polymers. Appl Phys Lett. 1990;57(8):765-767.

[66] Rebollar E, Vazquez de Aldana JR, Martin-Fabiani I, Hernandez M, Rueda DR, Ezquerra TA, et al. Assessment of femtosecond laser induced periodic surface structures on polymer films. Phys Chem Chem Phys. 2013;15(27):11287-11298.

[67] Rebollar E, Perez S, Hernandez M, Domingo C, Martin M, Ezquerra TA, et al. Physicochemical modifications accompanying UV laser induced surface structures on poly(ethylene terephthalate) and their effect on adhesion of mesenchymal cells. Phys Chem Chem Phys. 2014;16(33):17551-17559.

[68] Slepicka P, Rebollar E, Heitz J, Svorcik V. Gold coatings on polyethyleneterephthalate nano-patterned by F-2 laser irradiation. Appl Surf Sci. 2008;254(11):3585-3590.

[69] Pérez S, Rebollar E, Oujja M, Martín M, Castillejo M. Laser-induced periodic surface structuring of biopolymers. Appl Phys A. 2013;110:683-690.

[70] Bolle M, Lazare S. Large scale excimer laser production of submicron periodic structures on polymer surfaces. Appl Surf Sci. 1993;69(1-4):31-37.

[71] Niino H, Kawabata Y, Yabe A. Application of excimer laser polymer ablation to alignment of liquid crystals: Periodic microstructure on polyethersulfone. JAPANESE JOURNAL OF APPLIED PHYSICS. 1989;28.

[72] Lochbihler H. Colored images generated by metallic sub-wavelength gratings. Opt Express. 2009;17(14):12189-12196.

[73] Mate CM, Toney MF, Leach KA. Roughness of thin perfluoropolyether lubricant films: Influence on disk drive technology. IEEE Transactions on Magnetics. 2001;37(4 I):1821-1823. [74] Tsutsumi N, Fujihara A. Self-assembled spontaneous structures induced by a pulsed laser on a surface of azobenzene polymer film. J Appl Phys. 2007;101(3):033110-033115.

[75] Schwartzkopf M, Buffet A, Korstgens V, Metwalli E, Schlage K, Benecke G, et al. From atoms to layers: in situ gold cluster growth kinetics during sputter deposition. Nanoscale.

2013;5(11):5053-5062. 
[76] Rebollar E, Rueda DR, Martín-Fabiani I, Rodríguez-Rodríguez Á, García-Gutiérrez M-C, Portale G, et al. In Situ Monitoring of Laser-Induced Periodic Surface Structures Formation on Polymer Films by Grazing Incidence Small-Angle X-ray Scattering. Langmuir. 2015;31(13):3973-3981.

[77] Martín-Fabiani I, Rebollar E, García-Gutiérrez MC, Rueda DR, Castillejo M, Ezquerra TA. Mapping the Structural Order of Laser-Induced Periodic Surface Structures in Thin Polymer Films by Microfocus Beam Grazing Incidence Small-Angle X-ray Scattering. ACS Appl Mater Interfaces. 2015;7(5):3162-3169.

[78] Rebollar E, Sanz M, Perez S, Hernandez M, Martin-Fabiani I, Rueda DR, et al. Gold coatings on polymer Laser Induced Periodic Surface Structures: Assessment as substrates for Surface-Enhanced Raman Scattering. Phys Chem Chem Phys. 2012;14(45):15699-15705. [79] Rebollar E, Hernández M, Sanz M, Pérez S, Ezquerra TA, Castillejo M. Laser-induced surface structures on gold-coated polymers: Influence of morphology on surface-enhanced Raman scattering enhancement. Journal of Applied Polymer Science. 2015;132(45):n/a-n/a. [80] Bao Z, Dodabalapur A, Lovinger AJ. Soluble and processable regioregular poly(3hexylthiophene) for thin film field-effect transistor applications with high mobility. Appl Phys Lett. 1996;69(26):4108-4110.

[81] Kim DH, Park YD, Jang Y, Yang H, Kim YH, Han JI, et al. Enhancement of Field-Effect Mobility Due to Surface-Mediated Molecular Ordering in Regioregular Polythiophene Thin Film Transistors. Adv Func Mater. 2005;15(1):77-82.

[82] Zhang R, Li B, Iovu MC, Jeffries-El M, Sauve G, Cooper J, et al. Nanostructure dependence of field-effect mobility in regioregular poly(3-hexylthiophene) thin film field effect transistors. Journal of American Chemical Society. 2006;128(11):3480-3481.

[83] Sirringhaus H, Brown PJ, Friend RH, Nielsen MM, Bechgaard K, Langeveld-Voss BMW, et al. Two-dimensional charge transport in self-organized, high-mobility conjugated polymers. Nature. 1999;401(6754):685-688.

[84] Collins BA, Tumbleston JR, Ade H. Miscibility, Crystallinity, and Phase Development in P3HT/PCBM Solar Cells: Toward an Enlightened Understanding of Device Morphology and Stability. The Journal of Physical Chemistry Letters. 2011;2(24):3135-3145.

[85] Campoy-Quiles M, Ferenczi T, Agostinelli T, Etchegoin PG, Kim Y, Anthopoulos TD, et al. Morphology evolution via self-organization and lateral and vertical diffusion in polymer:fullerene solar cell blends. Nature Materials. 2008;7(2):158-164.

[86] Martínez-Tong DE, Rodríguez-Rodríguez Á, Nogales A, García-Gutiérrez M-C, PérezMurano F, Llobet J, et al. Laser Fabrication of Polymer Ferroelectric Nanostructures for Nonvolatile Organic Memory Devices. ACS Appl Mater Interfaces. 2015;7(35):19611-19618. 